Instituto Internacional de Investigación y Desarrollo Tecnológico Educativo INDTEC, C.A.

DOI: https://doi.org/10.29394/scientific.issn.2542-2987.2017.2.5.11.201-221

OAI-PMH: http://www.indteca.com/ojs/index.php/Revista Scientific/oai

\title{
La Comunicación Humana del Gerente en las Organizaciones del Siglo XXI
}

\author{
Autora: Olga Vargas Cordero \\ Universidad Centroccidental "Lisandro Alvarado", UCLA \\ olga.vargas@ucla.edu.ve \\ Lara, Venezuela
}

\section{Resumen}

El artículo ofrece los resultados de un estudio cuantitativo sobre la comunicación humana del gerente en las organizaciones del siglo XXI, que soportado en el principio epistémico de la complementariedad se aplicaron herramientas de coaching (Feedback $360^{\circ}$ y Rueda de la Vida) y entrevista no estructurada a seis gerentes de CORPOELEC, para develar cómo interacciona tanto horizontal como verticalmente e identificar barreras comunicacionales desde el elemento ontológico del gerente. Los hallazgos de la investigación señalan la presencia de barreras comunicativas culturales y psicosociales que impactan las interacciones (gerente-gerente superior, gerente-gerente y gerente-subordinado), por tal motivo, entre otros lineamientos se recomienda el Coaching Ontológico para desarrollar un gerente persona con competencias de comunicación humana, valoración personal y manejo de diferencias que humanice la comunicación en la organización del siglo XXI.

Palabras clave: comunicación humana; comunicación verbal; comunicación no verbal. 


\title{
The Manager's Human Communication in the Organizations of the XXI Century
}

\begin{abstract}
The article offers the results of a quantitative study on the manager's human communication in organizations of the $\mathrm{XXI}$ century, supported by the epistemic principle of complementarity, coaching tools $\left(360^{\circ}\right.$ Feedback and Wheel of Life) and unstructured interview to six managers of CORPOELEC, to reveal how it interacts both horizontally and vertically and to identify communication barriers from the manager's ontological element. The research findings point to the presence of cultural and psychosocial communication barriers that impact interactions (senior manager-manager, manager-manager and manager-subordinate). For this reason, among other guidelines, Ontological Coaching is recommended to develop a person manager with competences of human communication, personal valuation and management of differences that humanize the communication in the organization of the XXI century.
\end{abstract}

Keywords: human communication; verbal communication; non-verbal communication. 


\section{Introducción}

En la Sociedad del Conocimiento, en una organización del siglo XXI, el gerente es el emisor principal del proceso de comunicación organizacional, es quien activa el sistema nervioso comunicativo a través de los distintos mensajes o unidades de comunicación que emergen durante su práctica gerencial y que emite a los diferentes públicos con los cuales interacciona.

En efecto, en la vida organizacional tanto la comunicación como la gerencia van de la mano "difícilmente podemos imaginar una acción directiva sin comunicación o una comunicación exenta de dirección. También resulta difícil imaginar la construcción de un futuro sin acción directiva y sin acción comunicativa..." (Rojas, Arapé, Mújica, Rodríguez, Lara, Durand y Ugas, 1999: 57). Ciertamente, dentro de las organizaciones se convive y se gerencia en torno a la comunicación. Las organizaciones utilizan gerencialmente a la comunicación en sus dimensiones interpersonales, grupales, corporativas y sociales. Por lo tanto, la organización vive a partir de la comunicación y ésta se convierte en expresión de la organización.

Particularmente, al ubicar a ese homo humanus llamado directivo o gerente como una totalidad en el plano de una organización del siglo XXI, sea esta pública o privada, por su accionar y características inherentes a la praxis gerencial, emerge en él su necesidad de relacionarse con otros seres humanos, con quienes entreteje la red comunicacional organizacional, para codificar y decodificar las complejidades, incertidumbres, paradojas y globalidad de los procesos que se desarrollan en el ambiente laboral.

En estas relaciones comunicativas con otros individuos, todo lo que diga o haga el gerente en su organización comunica; la comunicación del gerente se mueve en distintos niveles desde lo intrapersonal (consigo mismo), interpersonal (con una persona), grupal (con más de dos personas), organizacional (con otros individuos y organizaciones), no obstante, de acuerdo a Bateson (citado en Castro y Moreno, 2006) a la comunicación 
interpersonal o la comunicación humana se le considera como la matriz de los demás niveles comunicativos.

Según Watzlawick, Beavin y Jackson (1985: 17) la comunicación humana es "...una condición sine qua non de la vida humana y el orden social...", en este sentido los referidos autores sostienen que toda conducta en una situación de interacción, tiene un valor de un mensaje, es decir, es comunicación actividad o inactividad, palabras o silencio, tiene siempre valor de mensaje, que no es posible no comunicarse.

Por tanto, el gerente al establecer una comunicación humana con sus públicos organizacionales, a través de estos flujos comunicativos gerenciales da conocer su estilo de gerencia y relación, forma de ser, valores, rasgos particulares, auto expresa su identidad, su self o sí mismo (Costa, 2009: 16); es decir, por medio de sus acciones comunicativas el directivo visibiliza sus competencias gerenciales estratégicas y comunicativas, se hace humano 0 inhumano ante los otros humanos.

De acuerdo con estos razonamientos y focalizados en un contexto organizacional venezolano emergen las siguientes preguntas que direccionan el abordaje de la presente investigación: ¿Por qué generar un constructo teórico de la comunicación humana del gerente? ¿Cómo el gerente venezolano interacciona con sus superiores, pares y subordinados en la praxis gerencial? ¿Qué barreras comunicativas emergen en el gerente venezolano en su proceso de interacción gerencial? ¿Qué elemento del gerente se debe tomar en consideración en su comunicación humana? ¿Qué lineamientos gerenciales se deben diseñar para que el gerente venezolano humanice su comunicación?

Como objetivo general de la correlación de las referidas interrogantes se planteó generar un constructo teórico de la Comunicación Humana del Gerente en las Organizaciones del Siglo XXI, así como los siguientes objetivos de investigación, que constituyó la teleología del estudio de carácter 
descriptivo, enmarcado dentro de un diseño no experimental, con un enfoque cuantitativo:

1. Develar cómo el gerente venezolano interacciona con sus superiores, pares y subordinados.

2. Identificar las barreras comunicativas que emergen en el gerente venezolano en su proceso de interacción gerencial.

3. Describir el elemento ontológico del gerente en la comunicación humana.

4. Proponer lineamientos para que el gerente humanice su comunicación en la praxis gerencial en las organizaciones del siglo XXI.

\section{Referentes Teóricos}

Para comprender la comunicación humana del gerente en la organización del siglo XXI, es necesario partir de la concepción epistemológica de la comunicación. En este sentido, en importante aclarar que, si bien los estudios de la comunicación han centrado su atención en varios objetos de estudio, la reflexión sobre los propios marcos teóricos, metodológicos y epistemológicos sobre los que se basa su práctica de investigación, no han sido objeto de reflexión suficiente, "no vemos que no vemos" según Maturana y Varela (citado por Vidales, 2011: 13). El fenómeno de la comunicación ha sido objeto de investigación de las diferentes disciplinas: psicología, sociología, antropología, ciencias políticas, entre otras, cada una de ellas ha concebido la comunicación desde su predominio, por lo cual en el ámbito teórico se encuentran una cantidad de conceptualizaciones y posturas sobre la comunicación, generando como resultado "...la polisemia misma del concepto de comunicación" (Rizo, 2011: 3). Por este motivo el concepto de comunicación se hace difícil enmarcar o delimitar cuando se trabaja como objeto de estudio. 


\subsection{Teoría de la Comunicación Humana de Paul Watzlawick}

La Teoría de la Comunicación Humana de Watzlawick y otros (1985), se deriva de los trabajos realizados en la Escuela de Palo Alto, que desde un enfoque sistémico, por un lado, delimita con claridad lo que se entiende por interacción comunicativa, fundamento básico para las relaciones humanas y, por el otro, basa su constructo teórico en los denominados Axiomas de la comunicación, a saber:

1. Es imposible no comunicar, por lo que en un sistema dado, todo comportamiento de un miembro tiene un valor de mensaje para los demás.

2. En toda comunicación cabe distinguir entre aspectos de contenido o semánticos y aspectos relacionales entre emisores y receptores.

3. La definición de una interacción está siempre condicionada por la puntuación de las secuencias de comunicación entre los participantes.

4. La comunicación humana implica dos modalidades: la digital, qué se dice (comunicación verbal) y la analógica, cómo se dice (comunicación no verbal).

5. Toda relación de comunicación es simétrica o complementaria, según se base en la igualdad o en la diferencia de los agentes que participan en ella, respectivamente.

De los referidos axiomas se desprende que el individuo es el único organismo que utiliza tanto los modos de comunicación digital (comunicación verbal) y analógicos (comunicación no verbal). La comunicación digital (comunicación verbal), es lo que dice la persona y se transmite a través de símbolos lingüísticos o escritos y es el vehículo del contenido de la comunicación.

Mientras tanto en la comunicación analógica (comunicación no verbal), el término incluye cualquier manifestación no verbal que el organismo sea capaz de emitir, esto envuelve posturas, gestos, expresión facial, inflexión de 
la voz, la secuencia, el ritmo y la cadencia de las palabras mismas y cualquier otra expresión no verbal del organismo, así como los indicadores comunicativos que inevitablemente aparece en cualquier contexto en que tiene lugar una interacción.

\subsection{Teoría Enfoque Centrado en la Persona de Carl Rogers}

Según Martínez (2006) el valor más grande de la Teoría Centrada en la Persona de Carl Rogers reside en haber captado un principio básico de la naturaleza íntima de todo ser vivo: la necesidad que tiene de un ambiente, una atmósfera, un clima propicio y adecuado, que cuando se le ofrecen (en el caso humano, a través de las características de la autenticidad, la aceptación incondicional y la comprensión empática), activan su tendencia actualizante, es decir, despliegan su máxima potencialidad de desarrollo y creatividad, llegan a niveles de excelencia difíciles de imaginar en esta evolución perenne de la vida, en general y de los humanos, en particular.

\subsection{Modelos de Comunicación de Virginia Satir}

El presente estudio se soportó en los modelos de comunicación de Satir (1980), la autora ha descrito cinco modelos de comunicación que exponen las formas de cómo se relacionan las personas. Particularmente, para la investigación se consideró el modelo de comunicación acusador-recriminador que caracteriza a una persona con una comunicación deshumanizada y el modelo de comunicación congruente-auténtico que presenta a un individuo con una comunicación motivadora y de respeto.

\subsection{Modelo de Desarrollo Humano de Manuel Barroso}

La investigación consideró el modelo de Desarrollo Humano de Manuel Barroso, quien parte de las necesidades y autoestima de la persona y del 
conocimiento como procesos medulares para el desarrollo de competencias personales, organizacionales, comunicacionales y de liderazgo, entre otros.

Barroso (1998) expone un sistema total compuesto por dos subsistemas, bien definidos y estructurados: el sí mismo y el contexto, los dos grandes componentes de la autoestima como principio organizador de la vida de las personas. El subsistema sí mismo que tiene como elemento estructural las necesidades, genera cuatro competencias fundamentales como producto de las distintas configuraciones que adopta la energía en el proceso de satisfacción de las mismas: valoración personal, manejo de riesgos, comunicación y manejo de diferencias, y el subsistema contexto (contexto individual, contexto de pareja, contexto de familia, contexto organizacional y contexto social), donde esas necesidades quedan insertas, única manera de ser manejadas. Ambos subsistemas están relacionados entre sí, no pudiendo el uno existir sin el otro.

\subsection{Barreras del Gerente en la Comunicación Interpersonal Efectiva}

Los gerentes se enfrentan a barreras que pueden distorsionar el proceso de comunicación humana, para Lucas (2009) estos obstáculos suelen ser comunes al emisor y al receptor, al respecto describe estas barreras comunicativas como: Culturales: En tanto que puede dificultar el mensaje por sus deficiencias, conceptualización del mismo, formulación, selección semántica de los términos empleados, inteligencia y comprensión y conocimiento del código de comunicación de la propia cultura. Personales: Manera de ser o estar, el talante o estilo personal, la falta de convicción, el rol desde el que se comunica o se recibe la comunicación, el marco de referencias y la imagen social. Psicosociales: clima psicológico desfavorable, el lenguaje rebuscado, inadecuado, experiencias y culturas muy diversas e intensidad de la proyección, motivación. 


\section{Metodología de la Investigación}

La investigación se enmarcó dentro de un diseño no experimental, puesto que se realizó sin la manipulación de la variable. Sobre este particular, Hernández, Fernández y Baptista (2006) refieren que en este tipo de estudio se recaba información tal cual como se producen los hechos, para su posterior análisis, lo que permitirá emitir alguna conclusión. De igual forma, la investigación se concibió de carácter descriptivo, en razón de que se interesó por las condiciones y relaciones comunicativas actuales del gerente en las organizaciones del siglo XXI. Asimismo, el estudio se abordó a través de un enfoque cuantitativo, el cual según Hernández y otros (2006) el logro de los objetivos planteados, se obtienen a través de análisis estadísticos de los datos recolectados, en virtud del establecimiento de patrones de comportamiento en la población estudiada.

\subsection{Población y Muestra}

De acuerdo a Balestrini (2003), la población o universo de estudio, puede estar referido a cualquier conjunto de elementos de los cuales se pretende indagar y conocer sus características, o una de ellas y para el cual serán válidas las conclusiones obtenidas, la investigación tuvo como población de estudio la Corporación Eléctrica Venezolana (CORPOELEC), específicamente la Unidad de Planificación de la Zona Capital y Vargas, conformada por sesenta (60) personas, (7 gerentes y 53 subordinados). Basada en Moreno (2000: 22) en cuanto a que en el muestreo opinático o intencional el investigador "...determina cuales son los elementos que integrarán la muestra confiando en que el grupo que ha seleccionado es representativo de la población", la investigadora tomó como muestra opinática a 6 gerentes que incluyó al gerente superior y a 10 subordinados. 


\subsection{Técnicas e Instrumentos de Recolección de Datos}

La comunicación humana del gerente en las organizaciones del siglo XXI se estudió con herramientas de coaching (Rueda de la Vida y Feedback $\left.360^{\circ}\right)$ complementadas con entrevista no estructurada, para esta última se realizó una guía de entrevista. Por medio de la Rueda de la Vida, el gerente valoró con una puntuación del 1 al 10, el grado de satisfacción de las ocho (8) áreas más importantes de la vida del ser humano, mientras tanto, el Feedback $360^{\circ}$ permitió evaluar la comunicación humana del gerente (comunicación verbal y comunicación no verbal).

Para el Feedback $360^{\circ}$ se generó un cuestionario autoadministrado que se particularizó para cada una de la fuentes, dando así cuatro (4) cuestionarios autoadministrados (instrumento de evaluación al gerente por su jefe, instrumento de evaluación al gerente por sus pares, el instrumento de evaluación al gerente por sus subordinados y el instrumento de autoevaluación del gerente), los cuales fueron entregados a las distintas fuentes que dieron el feedback al gerente (incluyendo el mismo gerente).

El cuestionario se estructuró en una primera parte que contuvo veinticinco (25) ítems de escala de respuesta tipo Lickert, orientado a medir la dimensión comunicación humana en las subdimensiones comunicación ascendente, comunicación horizontal y comunicación descendente tanto en la comunicación verbal como en la comunicación no verbal. La segunda, con veinticinco (25) ítems que permitieron relacionar la dimensión barrera comunicativa en las subdimensiones barrera cultural, barreras personales y barreras psicosociales, de igual forma se les ofreció libertad a los informantes de incluir información adicional, en cuanto a que mencionarán al menos tres (3) recomendaciones que a su juicio contribuirían a mejorar la praxis comunicativa del gerente en la organización.

El cuestionario se sustentó en la Teoría de la Comunicación Humana de Paul Watzlawick, la Teoría Enfoque Centrado en la Persona de Carl Rogers 
y los Modelos de Comunicación de Virginia Satir, asimismo se le aplicó la validación de los instrumentos que según Hernández y otros (2006: 277) "se refiere al grado que un instrumento realmente mide la variable que se pretende medir". A tal efecto, la técnica empleada fue la de juicio de expertos, para la cual se seleccionaron tres (3) expertos, es decir, dos (2) especialistas en Comunicación Organizacional y un (1) metodólogo, quienes a través de una matriz de validación marcada, emitieron sus opiniones y sugerencias, obteniendo la validez de los mismos.

El análisis de los datos se realizó través de la estadística promedio, particularmente para la categorización de la dimensión Comunicación Humana y Barreras Comunicativas, para la triangulación de la información de la data que emergió de las herramientas coaching (Feedback $360^{\circ}$ y Rueda de la vida) se empleó el Programa de Análisis Estructural del MIC-MAC (Matriz de Impactos Cruzados y una Multiplicación Aplicada a una Clasificación) y para el análisis de la entrevista no estructurada, definida por Méndez (2007) como una variante de entrevista, se aplicó el análisis de contenido.

\section{Resultados}

Objetivo 1: La Comunicación Humana del Gerente en las Organizaciones del Siglo XXI.

Cuadro 1. Comunicación Humana del Gerente Siglo XXI.

\begin{tabular}{|c|c|c|c|c|c|c|}
\hline \multirow[t]{2}{*}{ Subdimensiones } & \multicolumn{2}{|c|}{$\begin{array}{c}\text { Comunicación } \\
\text { Verbal }\end{array}$} & \multicolumn{2}{|c|}{$\begin{array}{l}\text { Comunicación } \\
\text { No Verbal }\end{array}$} & \multicolumn{2}{|c|}{ Porcentaje } \\
\hline & $\%$ & Categoría & $\%$ & Categoría & $\%$ & Categoría \\
\hline $\begin{array}{l}\text { Comunicación } \\
\text { Ascendente } \\
\text { (Superior) }\end{array}$ & 4.0 & Alta & 4.1 & Alta & 4.1 & Alta \\
\hline $\begin{array}{c}\text { Comunicación } \\
\text { Horizontal } \\
\text { (Pares) }\end{array}$ & 3.8 & Alta & 4.0 & Alta & 3.9 & Alta \\
\hline $\begin{array}{l}\text { Comunicación } \\
\text { Descendente } \\
\text { (Subordinados) }\end{array}$ & 3.9 & Alta & 4.1 & Alta & 4.0 & Alta \\
\hline Total & 3.9 & Alta & 4.0 & Alta & 3.9 & Alta \\
\hline & & & & cación Humana & 3.9 & Alta \\
\hline
\end{tabular}

Fuente: Vargas (2017) 
Los resultados muestran que la Comunicación Verbal y la Comunicación No Verbal constituyen la Comunicación Humana del gerente venezolano en la organización del siglo XXI, tal como lo exponen los promedios registrados para cada modo de comunicación. De igual forma, los informantes coinciden en que la comunicación humana de los gerentes en una organización se establece de forma ascendente (superior), horizontal (pares) y descendente (subordinados).

Entre los aspectos positivos se revela que el gerente venezolano de la organización del siglo XXI está en una comunicación verbal permanente en su praxis gerencial, que su superior valora su capacidad para comunicarse, así como los intercambios comunicativos con los gerentes pares son simétricos y equilibrados, de apoyo mutuo y a los subordinados le suministran de forma verbal información relacionada con el trabajo.

Sin embargo, es importante resaltar que en la comunicación verbal del gerente venezolano en las díadas gerente-gerente superior y gerentesubordinado, emerge que en las interacciones de la praxis gerencial puntúan mensajes incompletos, que se configuran como patrones de intercambios en los contenidos de los mensajes verbales, mientras con los gerentes pares no se presenta este fenómeno comunicativo.

Por su parte, el Indicador Comunicación No Verbal registró un promedio de 4.1, valorado en una categoría alta, con lo cual se evidencia que los gerentes en sus interacciones con sus superiores, gerentes pares y subordinados también se comunican de manera no verbal.

Entre los aspectos positivos de la comunicación no verbal del gerente venezolano con sus superiores, pares y subordinados resalta el uso de un tono de voz firme y equilibrado, con pausas y buena pronunciación, de igual forma expresa con firmeza, claridad y precisión sus ideas y opiniones, mientras habla mira directamente a los ojos de su interlocutor y sus palabras concuerdan tanto con su expresión facial como con la posición de su cuerpo. No obstante, 
también cabe destacar que en la comunicación no verbal del gerente venezolano se devela que hace un reducido uso del espacio interpersonal y de su estructuración (proxémia) con el superior y subordinados y, medianamente, con los gerentes pares.

Los hallazgos revelan que la comunicación humana de los gerentes de la organización del siglo XXI tiene un aspecto de contenido y un aspecto relacional, ambos modos de comunicación no solo existen lado a lado, sino que se complementan entre sí en cada mensaje, el aspecto relativo al contenido se transmite en forma digital (verbal), mientras que el aspecto relativo a la relación es de naturaleza predominante analógica (no verbal).

Objetivo 2: Identificación de las barreras comunicativas que emergen en el gerente venezolano en su proceso de interacción gerencial.

Los hallazgos revelan que más allá de las categorías altas, en la comunicación humana de los gerentes de la organización del siglo XXI emergen barreras comunicativas como las barreras culturales y psicosociales que obstaculizan las interacciones que se dan en la comunicación ascendentes (con su superior), comunicación horizontal (con sus pares) y comunicación descendentes (con sus subordinados), aunque los informantes en su develar, en apariencia pareciera que no hay, sin embargo, el análisis exhaustivo revela lo contrario. A continuación, se presenta un resumen de los hallazgos más relevantes del segundo objetivo:

- En las interacciones del gerente venezolano con sus jefes en la organización del siglo XXI, se revela como una barrera cultural los mensajes incompletos como patrón en los intercambios comunicativos.

- En las interacciones del gerente venezolano con sus pares gerentes en la organización del siglo XXI, se configura como barrera cultural que los directivos informan medianamente sobre los aspectos de la tarea laboral que han hecho bien. 
- En las interacciones de los gerentes con sus subordinados en la organización del siglo XXI sobresale como barrera psicosocial que motiva moderadamente al personal en función de su satisfacción laboral.

- En las interacciones del gerente venezolano en la organización del siglo XXI emerge como una barrera psicosocial que el directivo crea moderadamente un ambiente laboral agradable, humano, pacifico, acogedor, de apertura para una auténtica relación con los integrantes de la organización.

- En la comunicación del gerente venezolano con sus subordinados en la organización del siglo XXI se revela como barrera psicosocial que medianamente comparte planes, descubre y promueve las oportunidades de colaboración.

- El gerente venezolano de la organización del siglo XXI percibe que medianamente motiva al personal en función de su satisfacción laboral, opinión que coincide con la de sus subordinados.

Objetivo 3: Lo Ontológico del Gerente en la Comunicación Humana de la Organización del Siglo XXI.

Para describir el elemento ontológico del gerente y cómo se expresa a través de la comunicación humana, la aplicación de la herramienta Rueda de la Vida permitió que los gerentes desde su contexto organizacional revelaron su actual nivel de satisfacción con respecto a las necesidades (trabajo, dinero, salud, amigos/social, familia, pareja, espiritual y ocio), en relación al nivel de satisfacción que le gustaría obtener, con estos resultados emergió esta Rueda de la Vida de los gerentes de la organización del siglo XXI: 
Gráfico 1. Rueda de la Vida de los Gerentes de la Organización del Siglo XXI.

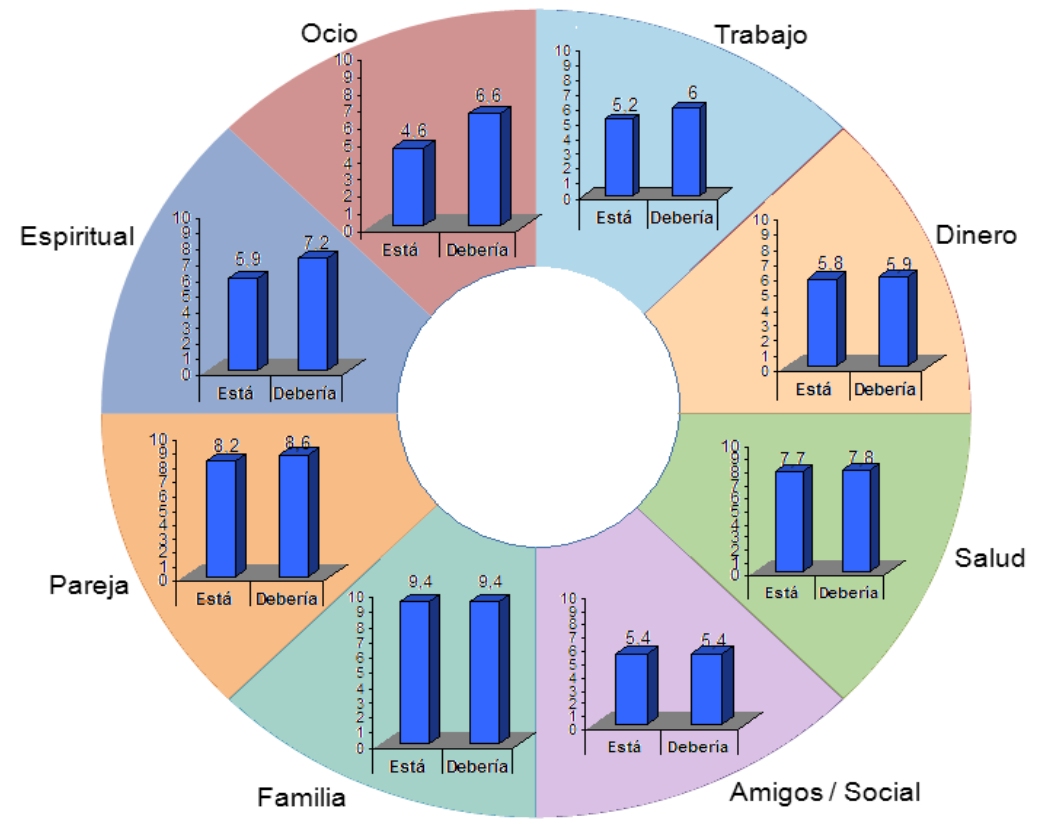
indirectas. Particularmente, cabe resaltar que entre los resultados de influencias indirectas muestra la influencia que la comunicación humana tiene 
sobre el homo gerente y a la vez como el homo gerente tiene una influencia en el sí mismo, de lo cual se desprende que un gerente que toma conciencia de sus propias necesidades y opera desde su interioridad, toma en cuenta las necesidades de los otros, de la organización, se ubica dentro de un contexto específico, y busca la congruencia de todos.

\section{Lineamientos para el Gerente Siglo XXI}

Con base en los resultados obtenidos en este estudio y de acuerdo a lo expresado por los gerentes y subordinados en el Feedback $360^{\circ}$, se proponen lineamientos para que el gerente humanice su comunicación en la praxis gerencial en la organización del Siglo XXI, de esta manera se da respuesta al último objetivo planteado en la presente investigación.

\subsection{Lineamiento General}

Considerar el Coaching Ontológico para desarrollar un gerente persona con competencias de comunicación humana.

\subsection{Lineamientos Específicos}

Se presentan lineamientos específicos para las diadas gerente-gerente superior, gerente-gerente y gerente-subordinado.

\subsubsection{Gerente-Gerente Superior}

Realizar plenarias motivacionales, sesiones de coaching, de igual forma efectuar encuestas de clima laboral en la organización para identificar problemas comunicacionales y solucionarlas.

Efectuar reuniones periódicas (mensuales) con la gerencia tanto para conocer lineamientos y comunicarlos a los subordinados, como realizar seguimiento y control de los planes que permitan retroalimentar los procesos, y que estas reuniones sostenidas estén sustentadas por minuta de trabajo. 
La Alta Gerencia se comunique asertivamente, con claridad, autenticidad y autoridad, que la emisión de las directrices y lineamientos sean más claros y que contenga todos los detalles de alcances, compromisos y metas.

\subsubsection{Gerente-Gerente (pares)}

Efectuar reuniones cara a cara, intercambios de información, rotación de gerentes, ser más abiertos.

Promover un clima de confianza para sus interlocutores y participar activamente en los eventos de acercamiento en la empresa.

Compartir en más oportunidades de forma extra laboral, interesarse en las aficiones y preferencias personales.

Mantener mayor relación en la organización con los pares y sus necesidades.

La transmisión del mensaje sea lo más completa posible, evitando las suposiciones y generalizaciones.

\subsubsection{Gerente-Subordinado}

Motivar a los subordinados mediante actividades de grupo, bien sea dinámicas grupales o de integración. Así como motivar al personal en función de su satisfacción laboral.

Mejorar cada día la interrelación con sus subordinados en busca de aumentar el clima laboral de la unidad.

Resaltar a los subordinados los aspectos positivos del trabajo realizado, así como sus habilidades humanas y de esta manera incentivar a que sus subordinados sean mucho más eficientes, eficaces y proactivos.

Transmitir información que llega de los altos niveles e Interpretar los lineamientos recibidos de forma tal que se puedan emitir de forma más completa. 


\section{Conclusiones}

La comunicación verbal y la comunicación no verbal constituyen la comunicación humana del gerente venezolano en la organización del siglo $X X I$, que establece en sentido ascendente, sentido horizontal y sentido descendente. En la praxis gerencial el trabajo de un directivo consiste fundamentalmente estar en interacción comunicativa.

En la comunicación verbal del gerente venezolano en las organizaciones del siglo XXI emerge que en sus interacciones con sus jefes $y$ subordinados puntúa mensajes incompletos que se configuran como patrones de intercambios en los contenidos de los mensajes verbales. Mientras en la comunicación no verbal emerge que no maneja los elementos proxémicos. Los superiores revelan que el gerente venezolano en su comunicación no verbal ascendente crea medianamente un ambiente laboral agradable y humano.

El estudio identificó barreras comunicativas culturales y psicosociales en los intercambios comunicativos de los gerentes en las díadas de comunicación ascendentes (jefes), comunicación horizontal (pares) y comunicación descendente (subordinados).

Se evidenció que un gerente satisfecho con sus necesidades, lo expresa a través de una comunicación humana congruente y efectiva, lo contrario se configura a través de comportamientos comunicativos que se reflejan en barreras $u$ obstáculos comunicativos (culturales, personales y psicosociales).

\section{Recomendaciones}

Se recomienda como lineamiento general el Coaching Ontológico para desarrollar un gerente persona con competencias de comunicación humana, valoración personal, manejo de riesgo y manejo de diferencias. Asimismo, se recomiendan lineamientos específicos orientados a cada una de las díadas 
que conforman el sistema interaccional de la organización del siglo XXI (gerente-gerente superior, gerente-gerente y gerente-subordinado).

Se recomienda tomar en consideración los lineamientos propuestos a fin de revisarlos, adaptarlos y ponerlos en práctica en función de fortalecer una comunicación humanista en las organizaciones del siglo XXI.

\section{Referencias}

Balestrini, M. (2003). Como se Elabora el Proyecto de Investigación. Consultores BL.

Barroso, M. (2005). Meditaciones Gerenciales. (Segunda reimpresión). Caracas, Venezuela: Galac.

Barroso, M. (1998). Autoestima: Ecología o Catástrofe. (Segunda edición). Caracas, Venezuela: Galac.

Castro L., I. y Moreno B., L. (2006). El Modelo Comunicativo: teóricos y teorías relevantes. (Primera edición). México: Trillas.

Costa, J. (2009). Identidad Corporativa. (Primera edición). México.

Hernández, R. Fernández, S., y Baptista, P. (2006). Metodología de la Investigación. (Cuarta edición). México, D.F.: Mc Graw Hill Interamericana Editores S.A. de C.V.

Lucas, A. (2009). La Nueva Comunicación. Madrid: Trotta.

Martínez, M. (2012). El Paradigma Emergente: hacia una nueva teoría de la racionalidad científica. (Segunda edición). (Reimpresión, 2012). México: Trillas, 1997.

Méndez, A. (2007). Metodología y técnicas de investigación aplicadas a la comunicación. Primera Edición. Colección Textos Universitarios. Ediciones del Vice Rectorado Académico Universidad del Zulia. Venezuela: Producciones Editoriales C.A.

Moreno, M. (2000). Introducción a la Metodología de la Investigación Educativa 2. 
Rojas, L., Arapé, E., Mújica, M., Rodríguez, R., Lara, C., Durand, E., Ugas, G. (1999). Comunicación, Gerencia y Futuro: Una interpretación Postmoderna. Revista Venezolana de Gerencia, Año 4. №9, 1999, 5169. Vicerrectorado Académico, LUZ. Caracas, Venezuela.

Rizo, M. (2011). Reseña de Teoría de la comunicación humana de Paul Watzlawick. Razón y Palabra, núm. 75. Recuperado de: http://www.redalyc.org/articulo.oa?id=199518706028

Satir, V. (1980). En contacto íntimo. Cómo relacionarse con uno mismo y con los demás. Ciudad de México: Concepto.

Vidales, C. (2011). Semiótica y Teoría de la Comunicación. Tomo II. Primera edición: marzo de 2011, Colección. Altos Estudios №. 24. Impreso en Monterrey, N. L., México.

Watzlawick, P., Beavin, J. y Jackson, D. (1985). Teoría de la Comunicación Humana. Interacciones, patologías y paradojas. (Cuarta edición). Barcelona: Herder. 


\section{Olga Vargas Cordero \\ e-mail: olga.vargas@ucla.edu.ve}

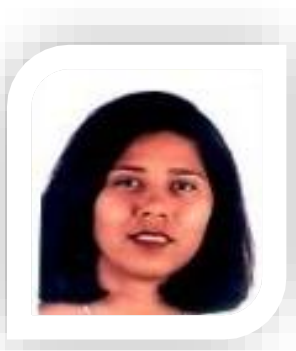

Nacida en Venezuela. Licenciada en Comunicación Social de la Universidad del Zulia (LUZ). Doctorada en Gerencia Avanzada de la Universidad Fermín Toro (UFT), con Maestría en Comunicación Corporativa (UFT), Diplomado en Docencia Universitaria (UCLA), Diplomado en Componente Docente (UFT), Diplomado en Administración de Módulos Instruccionales Asistidos por el Computador-MIAC (UFT), Diplomado en Comunicación Corporativa (UFT) y Diplomado en Gestión de la Comunicación Política y Electoral (UAB). Docente del Decanato de Ciencias y Tecnología de la Universidad Centroccidental "Lisandro Alvarado" (UCLA), en las cátedras Lenguaje y Comunicación, Lenguaje y Comunicación Estratégica para gerentes de Producción, actualmente integra la Comisión de Equivalencias del DCyT. 